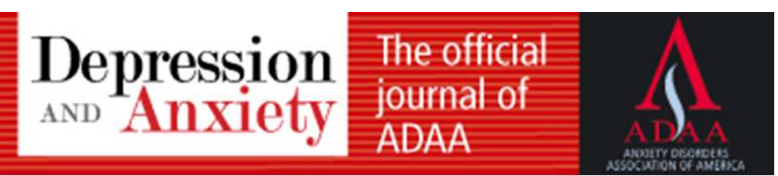

\title{
CBT and positive psychology interventions for clinical depression promote healthy attentional biases: An eye- tracking study
}

\begin{tabular}{|r|l|}
\hline Journal: & Depression and Anxiety \\
\hline Manuscript ID & DA-17-638.R1 \\
\hline Wiley - Manuscript type: & Research Article \\
\hline Complete List of Authors: & $\begin{array}{l}\text { Vazquez, Carmelo; Complutense University, School of Psychology } \\
\text { Duque, Almudena; Universidad Pontificia de Salamanca, School of } \\
\text { Psychology } \\
\text { Blanco, Ivan; Complutense University, School of Psychology } \\
\text { Pascual, Teodoro; Complutense University, School of Psychology } \\
\text { Poyato, Natalia; Complutense University, School of Psychology } \\
\text { Lopez-Gomez, Irene; Universidad Rey Juan Carlos, School of Psychology } \\
\text { Chaves, Covadonga; Universidad Francisco de Vitoria }\end{array}$ \\
\hline Keywords: & $\begin{array}{l}\text { Depression, Cognition, CBT/cognitive behavior therapy, dysthymic } \\
\text { disorder, treatment }\end{array}$ \\
\hline
\end{tabular}

\section{SCHOLARONE \\ Manuscripts}


CBT and positive psychology interventions for clinical depression promote healthy attentional biases: An eye-tracking study

\author{
Carmelo Vazquez ${ }^{\mathrm{a}}$, Almudena Duque ${ }^{\mathrm{b}}$, Ivan Blanco ${ }^{\mathrm{a}}$, Teodoro Pascual ${ }^{\mathrm{a}}$, Natalia \\ Poyato $^{\mathrm{a}}$, Irene Lopez-Gomez ${ }^{\mathrm{c}}$, and Covadonga Chaves ${ }^{\mathrm{d}}$ \\ ${ }^{a}$ School of Psychology, Complutense University of Madrid, Madrid, Spain \\ ${ }^{b}$ Pontifical University of Salamanca, Salamanca, Spain \\ ${ }^{c}$ Rey Juan Carlos University, Madrid, Spain \\ ${ }^{d}$ Francisco de Vitoria University, Madrid, Spain
}

Running Head: Attentional changes in psychotherapy

Word count: 3443

Keywords: depression; cognition; CBT/cognitive behavior therapy; dysthymic disorder; treatment

Address correspondence to:

Carmelo Vázquez

School of Psychology

Complutense University of Madrid

Campus de Somosaguas

Madrid, Spain, 28223

Email : cvazquez@ucm.es

Phone : (34) 91-3943090

FAX : (34) 91-3943189 


\begin{abstract}
Background: Although there is a growing interest in the role of attentional biases in depression, there are no studies assessing changes in these biases after psychotherapeutic interventions.

Methods: We used a validated eye-tracking procedure to assess pre-post therapy changes in attentional biases towards emotional information (i.e., happy, sad and angry faces) when presented with neutral information (i.e., neutral faces). The sample consisted of 75 participants with major depression or dysthymia. Participants were blindly assigned to one of two 10 weekly sessions of group therapy: a CBT intervention $(\mathrm{N}=41)$ and a Positive Psychology Intervention $(\mathrm{N}=34)$.
\end{abstract}

Results: Both treatments were equally efficacious in improving depressive symptoms $\left(p=.0001, \eta^{2}=.68\right)$. A significant change in attentional performance after therapy was observed irrespective of the intervention modality. Comparison of pre-post attentional measures revealed a significant reduction in the total time of fixations (TTF) looking at negative information (i.e., sad and angry faces) and a significant increase in the TTF looking at positive information (i.e., happy faces) -all $p$-values $>.02$.

Conclusions: Findings reveal for the first time that psychotherapeutic interventions are associated with a significant change in attentional biases as assessed by a direct measure of attention. Furthermore, these changes seem to operate in the same direction typically found in healthy populations (i.e., a bias away from negative information and a parallel bias towards positive information). These findings illustrate the importance of considering attentional biases as clinical markers of depression and suggest the viability of modifying these biases as a potential tool for clinical change. 


\section{Introduction}

The role that attentional biases play in depression has received increased attention in theoretical models of the disorder (Disner, Beevers, Haigh \& Beck, 2011). These biases have been investigated across various experimental paradigms. Using the emotional Stroop task, it has been observed that depressed participants show interference effects for both negative and positive words (Epp, Dobson, Dozois, \& Frewen, 2012; Peckham, McHugh \& Otto, 2010). Likewise, dot-probe tasks have demonstrated that depressed patients show a consistent attentional bias toward negative words (Donaldson, Lam \& Mathews, 2007) and negative pictures (Fritzsche et al., 2010) as well as a bias away from positive stimuli (Winer \& Salem, 2016).

Yet, standard assessment procedures of proxy measures of attention based on reaction times, such as those indexed in the Stroop task or the dot-probe, have been criticized because they do not allow investigators to explore the unique mechanisms underlying different stages of attentional processing (Yiend \& Mathews, 2004). Moreover, their reliability has also been questioned (Waechter, Nelson, Wright, Hyatt, \& Oakman, 2014; Vazquez, Blanco, Sanchez, \& McNally, 2016). An alternative to these procedures is eye-tracking methodologies. Using this approach, researchers have found that depressed participants fixate their gaze longer on negative stimuli than control participants (Caseras, Garner, Bradley \& Mogg, 2007; Leyman, De Raedt, Vaeyens, \& Phillipaerts, 2011). Interestingly, these biases have only been observed in the late and controlled components of attention (e.g., total fixation time), but not in early and automatic components (e.g., direction of initial gaze) - Armstrong \& Olatunji (2012). In other words, it seems depressed individuals have difficulties disengaging their gaze from negative stimuli once the negative stimuli have captured their attention. 
Additionally, depressed individuals also have difficulties engaging their attention towards positive information (Duque \& Vazquez (2015).

Most of the extant evidence on attentional biases in clinical depression is limited to cross-sectional studies. Therefore, research focused on analyzing changes in attentional biases after treatments for depression may clarify whether or not these biases are a stable characteristic associated with the disorder as well as their relationship with depression symptoms. Some studies have analyzed changes in attentional performance before and after cognitive therapy for depression using reaction time tasks or clinical neuropsychological tests (Segal \& Gemar, 1997; Groves et al., 2015). However, at present, there are no studies in which attentional patterns to emotional information have been directly assessed using an eye-tracking paradigm following psychological interventions for clinical depression. In the current study, we explored changes in attentional patterns to emotional information (i.e. emotional faces) in a sample of clinically depressed patients under one of two different treatments for depression: CBT vs. positive psychology interventions (PPI).

CBT is the most widely researched psychological treatment for depression (Hofmann, Asmudson \& Beck, 2013). It is recommended in treatment guidelines and the evidence supporting its efficacy for treating depression is strong (Cuijpers et al., 2011; Moriana, Gálvez-Lara \& Corpas, 2017). The main objective of CBT for depression is to challenge the content of negative thoughts, address cognitive biases in the processing of emotional information, and alleviate depressive symptoms and deficits.

PPI are a theoretically-grounded and empirically-based promising approach to increase positive emotions and psychological well-being (Lomas, Hefferon \& Ivtzan, 2016) which are considered critical psychopathological features of depression (Watson 
\& Naragon-Gainey, 2010). Meta-analytic evidence has shown that PPI are effective at decreasing depressive symptoms and increasing well-being (Bolier et al., 2013; Sin \& Lyubomirsky, 2009).

The main objective of our study was to explore changes in attentional patterns to emotional stimuli (i.e., happy, sad and angry faces) in clinically depressed individuals by comparing their attentional performance before and after receiving CBT or PPI to treat their depression. Previous research comparing the effects of CBT and PPI for depressed patients has shown significant changes in self-reported cognitive and wellbeing variables following both interventions (Chaves, Lopez-Gomez, Hervas \& Vazquez, 2017).

Our hypotheses were based on previous literature that has shown attentional biases are observed only in the late and controlled components of attention (i.e., total fixation time - Kellough, Beevers, Ellis \& Wells, 2008). The first hypothesis was that pre-post changes in attentional patterns associated with CBT or PPI would be significant in the maintenance of gaze index but not in the orienting index. Our second hypothesis was that, compared to pre-treatment assessment, depressed patients would show a significant reduction of maintenance of attention on negative stimuli (i.e., sad and angry faces) after CBT and PPI. Our third hypothesis was that, compared to pretreatment assessment, depressed patients would show an increase of maintenance of attention towards positive stimuli (i.e., happy faces) after CBT and PPI. Finally, we hypothesized that CBT would be more effective than PPI in reducing an attentional bias towards negative stimuli whereas PPI would more effective than CBT in increasing a bias towards positive stimuli. 


\section{Materials and Methods}

\subsection{Participants}

One hundred and twenty-eight depressed women who sought psychological treatment in a women's community center were included in the study (Lopez-Gomez, Chaves, Hervas \& Vazquez, 2017). Participants were blindly assigned to one of the interventions and assessors were not informed of each participant's treatment assignment. The flow chart provided in Figure 1 illustrates the study design. The final sample was composed of seventy-five women with a DSM-IV-TR diagnosis of major depressive disorder or dysthymia (APA, 2000). In the end, the CBT group consisted of 41 women, while the PPI group was composed of 34 women. The mean age of the sample was $51.08(S D=9.68)$. Exclusion criteria for the study were: substance abuse or dependence disorder (present), manic or hypomanic episodes (past or present), psychotic disorder (past or present), and a cognitive status (e.g., dementia or intellectual disability) that might prevent participants from following the interventions appropriately. All participants had normal or corrected vision through glasses or contact lenses.

\subsection{Intervention groups for depression}

Both intervention options consisted of 10 weekly, 2 hour sessions in a group format. Five groups for each condition, with a maximum of 15 participants per group, were included in the study. Two clinical psychologists administered both forms of intervention. Each session of the PPI and CBT programs had the same structure. They would begin with a review of the prior session's homework that was followed by an introduction of the topic of the day. Participants received guidance on how to carry out 
in-session exercises and therapists then provided the homework assignment for the next session.

The CBT intervention was an adaptation of the Group Therapy Manual for Cognitive-Behavioral Treatment of Depression (Muñoz, Aguilar-Gaxiola \& Guzman, 1995), based on the Coping with Depression course (Lewinsohn, Antonuccio, Breckenridge \& Teri, 1984). The PPI were a manualized protocol composed of empirically-validated positive psychology interventions for depression called Integrative Positive Psychological Intervention for Depression (IPPI-D) - (Chaves et al., 2017). None of the modules found in the intervention packages were aimed at modifying the focus of attention nor did they provide any kind of attentional training.

\subsection{Clinical Measures}

Structured Clinical Interview for DSM-IV (SCID-I, First, Spitzer, Gibbon, \& Williams, 1995). The SCID was used to assess the presence of major depressive disorder or dysthymia. The instrument was administered by trained clinical psychologists with extensive experience in structured diagnostic interviews.

Beck Depression Inventory-II (BDI-II; Beck, Steer, \& Brown, 1996). This is a 21-item self-report measure assessing depressive symptomatology. Each item consists of 4 statements which are ranked from 0 to 3 indicating symptom severity. The reliability in our study was high $(\alpha$ pre-treatment $=.88 ; \alpha$ post-treatment $=.93)$.

\subsection{Eye-tracking paradigm}

Stimuli. The stimuli were pairs of photographs, from the Karolinska Directed Emotional Faces (KDEF, Lundqvist, Flykt \& Öhman, 1998), with an actor/actress displaying different frontal view facial expressions in an emotional-neutral expression 
combination. A set of 28 faces (14 women, 14 men) were drawn for each emotional category: happiness, anger and sadness. Facial expressions were transformed into grayscale and framed with an oval window removing neck and hair from the original pictures (Calvo \& Lunqvist, 2008).

Equipment. A Tobii T120 eye tracker was used to measure the coordinates of participants' eye position every $16.7 \mathrm{~ms}$ (60 hertz). Ocular movements lasting more than 100 milliseconds (ms) within a visual angle of $1^{\circ}$ degree were classified as fixations. Tobii Studio 2.0.6 software was used for the presentation of the stimuli and collection of the attentional data. Two areas of interest (AOI) were defined for each trial. They corresponded to the total surface area for the emotional and neutral faces. This attention task has been previously used in similar studies (e.g., Sanchez, Vazquez, Gomez, \& Joormann, 2014).

\subsection{Procedure}

Once participants gave their written consent, the SCID diagnostic interview was administered. Participants who met inclusion criteria were selected for the study and assigned to one of the two treatment groups. One week before treatment started, participants completed the BDI-II and the attentional task.

The attentional task consisted of 84 trials, 28 trials for each emotional category: happiness, anger and sadness. Pairs of faces were shown in a $53 \mathrm{~cm}$ (width) x $30 \mathrm{~cm}$ (high) computer screen. The location in the screen of each facial expression (emotional and neutral) was counterbalanced and all were presented equally as often on the left as on the right side of the screen. Participants were seated in an anatomic chair at $73 \mathrm{~cm}$ from the screen, representing a horizontal visual angle of $14^{\circ}$ between the two pictures. 
Each trial started with a black screen for $500 \mathrm{~ms}$. Then, a white fixation cross was presented on the center of the screen for $500 \mathrm{~ms}$. After that, a random number between 1 and 9 appeared in the same central position for $1000 \mathrm{~ms}$. Participants were instructed to pronounce it aloud as quick as possible. This procedure has been used in previous studies (Calvo \& Avero, 2005; Vazquez et al., 2016) with the aim of ensuring the central position of the gaze before the appearance of the target stimuli. Immediately afterwards, a couple of facial expressions (happy vs. neutral, anger vs. neutral, sad vs. neutral) were presented for $3500 \mathrm{~ms}$. Participants were instructed to view the images naturally, with no further requirements. This free-viewing attentional task took approximately 10 minutes. To conclude this experimental session, each participant completed the BDI-II.

An identical experimental session was repeated one week after completion of each treatment program (10 sessions over the span of 2.5 months, approximately), as well as the diagnostic interview and the completion of the BDI-II.

\subsection{Dependent variables}

Two attentional indices were extracted for each participant. Direction of initial gaze (orienting index) is faster and automatic, as it reflects the early stages of visual attention processing, while total fixation time (maintenance index) is considered more controlled and to occur later in time.

a) Direction of initial gaze (i.e. location of the first fixation on one of the two facial expressions).

b) Total fixation time (i.e. total time that each subject fixated on each of the two facial expressions). 
Following the Shane and Peterson (2007) guideline, relative bias scores were calculated for each emotional category (happiness, anger and sadness). For direction of initial gaze index, we calculated the percentage of trials in which each participant initially fixated first on the emotional expression instead of the neutral one. The values obtained above $50 \%$ indicated the presence of a bias towards the emotional face (in the corresponding emotional category), while the values below 50\% indicated the presence of a bias toward the neutral one. Regarding total fixation time, relative bias scores were calculated by subtracting the obtained value of the neutral facial expression from the corresponding emotional expression value. Thereby, positive scores (greater than 0) were interpreted as the presence of bias towards emotional expressions. Conversely, negative scores (lower than 0 ) reflected a bias towards neutral expressions.

\subsection{Data analysis plan}

A 2 (Group: CBT, PPI) $\times 2$ (Time: pre-treatment, post-treatment) mixed-model analysis of variance (ANOVA) was conducted to analyze the effect of both treatments on depressive symptoms. Bonferroni follow-up tests were used to further analyze significant interactions.

Then, a series of 2 (Group: CBT, PPI) $\times 2$ (Time: pre-treatment, post-treatment) $\times 3$ (Emotional category: happy, angry, sad) mixed-model analysis of variance (ANOVA) was conducted to explore the effect of both treatments on attentional indices to emotional information. Bonferroni follow-up tests were used to further analyze significant interactions. Correlation analyses were performed to analyze the associations between severity of depression and attentional indexes.

\section{Results}




\subsection{Group characteristics}

As shown in Table 1, there were no significant differences between groups neither in demographic nor clinical characteristics.

\subsection{Changes in depressive symptoms severity}

Analysis showed a non-significant group $\times$ time interaction, $F(1,73)=1.26, p=$ $.26, \eta^{2}=.017$. Nonetheless, a significant main effect of Time $F(1,73)=152.98, p=$ $.0001, \eta^{2}=.68$ emerged (which indicated a large size effect associated with the interventions). Analyses revealed that both groups reported a significant decrease of depressive symptoms after treatment (Figure 2). Furthermore, there were no differences between treatments in regard to the clinical significance of changes as, at the end of the trial, only $21.9 \%$ of participants in the CBT group and $23.5 \%$ of participants in the PPI group still fulfilled diagnostic criteria of dysthymia or major depressive disorder, $X^{2}(1$, $n=75)=.03, p=.87$.

\subsection{Changes in attentional processing of emotional information}

a) Direction of initial gaze. Analyses showed a non-significant three-way interaction, $F(2,72)=.68, p=.51, \eta^{2}=.02$. However, a significant main effect of emotional category emerged, $F(2,72)=34.68, p<=.001, \eta^{2}=.49$, which was qualified by a significant two-way interaction, group $\times$ emotional category, $F(2,72)=5.57, p=$ $.006, \eta^{2}=.13$. Bonferroni post-hoc analyses revealed that both groups showed a greater initial frequency of directing their first fixation to happy faces than to sad (both $p<$ .001 ) or angry faces (both $p<.004$ ). Additionally, the CBT group, but not the PPI group, showed a greater tendency to fixate on angry faces than on sad ones $(p=.03)$. Regarding differences between groups, analyses revealed that, in comparison with the CBT group, PPI patients showed a higher tendency to fixate on sad faces $(p=.02)$. To explore this difference between groups we compared sad faces bias scores of each group 
with a no-bias criterion (50\%). Analyses showed a significant bias to sad faces in the PPI group, $t(33)=2.67, p=.01, d=.46$, but not in the CBT group $t(40)=-.60, p=.55$, $d=.09$. Finally, no effect of treatment was found in the direction of initial gaze index.

b) Total fixation time. Analysis showed a non-significant three-way interaction, $F(2,72)=.31, p=.73, \eta^{2}=.01$. However, a significant main effect of emotional category, $F(2,72)=7.58, p=.001, \eta^{2}=.17$, and a significant main effect of time, $F(1$, $73)=7.29, p=.009, \eta^{2}=.09$, emerged. These main effects were qualified by a significant emotional category $\times$ time interaction, $F(2,72)=3.31, p=.04, \eta^{2}=.08$. Bonferroni post-hoc analyses revealed significant changes in the processing of emotional faces after treatment in both groups. Participants of both groups showed a significant decrease in the total time that they spent looking at angry $(p=.02)$ and sad faces $(p=.003$; Figure 3$)$. Furthermore, after treatment both groups spent significantly more time looking at happy faces than looking at angry $(p<.001)$ and sad faces $(p$ $<.001)$. To identify the existence of objective attentional biases, apart from group differences, we compared the attentional bias scores to happy, sad and angry faces with a no-bias criterion (zero). Our results revealed a significant attentional bias towards happy faces after the treatment, $t(74)=5.62, p<.001, d=.65$, and a significant attentional bias away from angry, $t(74)=-2.48, p=.01, d=.29$, and sad expressions, $t$ (74) $=-2.04, p=.04, d=.24$, after the treatment.

\subsection{Attentional biases and changes in depressive symptoms}

A series of bivariate correlation analyses were conducted to explore whether changes in attentional bias were associated with changes in the severity of depressive symptoms. Table 3 shows the zero-order correlation coefficients. Analyses revealed that improvement in depressive symptoms was significantly correlated with attentional 
changes consisting of spending more time looking at happy faces and less time looking at angry and sad faces. Duration of initial fixations were not found to be significantly associated with clinical changes.

\section{Discussion}

There are few studies that have analyzed attentional biases in clinically depressed participants using eye-tracking methodologies (Armstrong \& Olatunji, 2012; Peckham et al., 2010). As far as we know, there is no previous study that has explored pre-post therapy changes in attentional patterns using this methodology in a clinical sample.

Regarding the first hypothesis, we expected that changes in attentional biases would be significant in relation to maintenance of gaze but not in orienting of attention. In line with this hypothesis, direction of initial gaze did not show significant changes associated solely with the time of assessment (i.e., pre- and post-intervention assessments) nor in its interaction with the intervention modality. Yet, there was a significant group x emotional category interaction. Participants in the PPI group, compared to participants in the CBT group, showed a significantly higher tendency to look first at sad faces when presented with neutral ones. As there were no time-based changes associated with this pattern, the results do not seem to be linked to the effects of the therapies. In fact, the tendency to look at sad faces vs. neutral faces was significantly larger in the PPI group than in the CBT group before the therapy and it remained the same after the intervention.

The results of the study fully confirmed our second and third hypotheses, which were related to maintenance index. Our second hypothesis was that, after interventions, participants would show a decrease of attention towards negative information (i.e., sad 
and angry faces) whereas the third hypothesis was that participants would exhibit an increase in attention towards positive information (i.e., happy faces) following treatment. As it is shown in Figure 3, both interventions significantly reduced the amount of time that participants spent looking at negative faces and increased the amount of time they engaged with positive faces. Therefore, the interventions were effective at changing the double attentional biases to emotional information that are typically found in people with depression (Duque \& Vazquez, 2015) in a direction similar to the "protective biases" that have been consistently observed in healthy participants (Hilt \& Pollak, 2013; McCabe, Gotlib \& Martin, 2000) helping them to regulate negative emotions (Sanchez, Joormann, Gomez \& Vazquez, 2014). This finding provides support for the need of incorporating positive aspects of functioning in the research of psychopathology (Vazquez, 2017) and supports the principle idea of anhedonia theories that depression must be characterized not only by the presence of negative emotions and cognitions but also by difficulties in experiencing or maintaining positive emotional states (Rømer Thomsen, Whybrow \& Kringelbach, 2015; Watson \& Naragon-Gainey, 2010).

Changes in patterns of maintenance of gaze towards positive and negative stimuli were not significantly affected by the modality of intervention, which disconfirmed our fourth hypothesis. Although CBT and PPI have both been shown to be efficacious at treating depression (Chaves et al., 2017), given their large differences in content and focus of the interventions, we expected that they might affect patients' attentional performance in a different manner (i.e., CBT would reduce the bias towards negative information more than PPI, whereas PPI would increase a bias towards positive information more than CBT). More research is clearly needed, but it is possible that attentional changes are an intermediate mechanism of some other 
underlying processes (e.g., improvements in executive functioning) that may take place during treatment (DeRubeis, Siegle \& Hollon, 2008; Roiser, Elliott \& Sahakian, 2012);

Our study has some strengths and limitations. Regarding its strengths, this is the first study of clinical depression directly measuring attention by using eye-tracking methodology and a robust experimental paradigm to assess cognitive biases in depression (Duque \& Vazquez, 2015; Sanchez et al., 2013). Also, the study includes participants with a clinical diagnosis of depression or dysthymia who have mean scores of depression in the range of 'severe depression'.

The study also has some limitations. The study did not assess any other type of cognitive performance (e.g., memory, interpretation, etc.) that may also be associated with changes in attention (Everaert, Koster \& Derakshan, 2012). Furthermore, all participants were women and the study did not include follow-up data, which might have been relevant to know if attentional changes persist over time Furthermore, systematic differences between dysthymia and major depression should be explored in future studies (such as differences in length or intensity of depressive symptoms) as they may affect attentional performance..

Finally, our results offer relevant contributions that may help the field better understand the nature of cognitive changes in therapeutic interventions (Cristea et al., 2015; Lorenzo-Luaces, German \& DeRubeis, 2015). The fact that significant clinical changes were accompanied by significant changes in attentional biases may reflect that attentional processes are a key ingredient of change. However, the precise role of attentional processes and how they influence and interact with other cognitive processes, symptoms, and neurobiological factors is still not well known (Disner et al., 2011; Roiser et al., 2012). As Zinbarg et al. (2010) stated, research on the correlates and 
mechanisms of successful treatments is still in its infancy. We hope that our study sheds light on the attentional mechanisms involved in symptom changes in depression. 


\section{Acknowledgements}

This research was partially supported by the Spanish Ministry of Economy (MINECO) grants (PSI2015-69253-R, PSI2014-61764-EXPLORA, PSI2014-56303-REDT, and BES-2013-064936), the Spanish Ministry of Education, Culture and Sports (FPU12/02342 and FPU13/05467), and UCM grant CT27/16. The authors want to thank Matthew Abrams for his comments on earlier versions of this manuscript. 


\section{References}

Armstrong, T., \& Olatunji, B. O. (2012). Eye tracking of attention in the affective disorders: A meta-analytic review and synthesis. Clinical Psychology Review, 32, 704-723.

Beck, A. T., Steer, R. A., \& Brown, G. K. (1996). Manual for the Beck Depression Inventory. San Antonio, TX: The Psychological Corporation.

Bolier, L., Haverman, M., Westerhof, G. J., Riper, H., Smit, F., \& Bohlmeijer, E. (2013). Positive psychology interventions: a meta-analysis of randomized controlled studies. BMC Public Health, 13, 119. doi:10.1186/1471-2458-13-119

Calvo, M. G., \& Avero, P. (2005). Time course of attentional bias to emotional scenes in anxiety: Gaze direction and duration. Cognition \& Emotion, 19, 433-451

Calvo, M. G., \& Lundqvist, D. (2008). Facial expressions of emotion (KDEF): Identification under different display-duration conditions. Behavior Research Methods, 40, 109-115.

Caseras, X., Garner, M., Bradley, B. P., \& Mogg, K. (2007). Biases in visual orienting to negative and positive scenes in dysphoria: An eye movement study. Journal of Abnormal Psychology, 116, 491-497.

Chaves, C., Lopez-Gomez, I., Hervas, G., \& Vazquez, C. (2017). A comparative study on the efficacy of a positive psychology intervention and a cognitive behavioral therapy for clinical depression. Cognitive Therapy and Research, 41, 417-433.

Cristea, I. A., Huibers, M. J., David, D., Hollon, S. D., Andersson, G., \& Cuijpers, P. (2015). The effects of cognitive behavior therapy for adult depression on dysfunctional thinking: A meta-analysis. Clinical Psychology Review, 42, 62-71. 
Cuijpers, P., Clignet, F., van Meijel, B., van Straten, A., Li, J., \& Andersson, G. (2011). Psychological treatment of depression in inpatients: a systematic review and meta-analysis. Clinical Psychology Review, 31, 353-360.

DeRubeis, R. J., Siegle, G. J., \& Hollon, S. D. (2008). Cognitive therapy vs. medications for depression: Treatment outcomes and neural mechanisms. Nature Reviews Neuroscience, 9, 788-796.

Disner, S. G., Beevers, C. G., Haigh, E. A., \& Beck, A. T. (2011). Neural mechanisms of the cognitive model of depression. Nature Reviews Neuroscience, 12, 467-477.

Donaldson, C., Lam, D., \& Mathews, A. (2007). Rumination and attention in major depression. Behaviour Research and Therapy, 45, 2664-2678.

Duque, A., \& Vázquez, C. (2015). Double attention bias for positive and negative emotional faces in clinical depression: Evidence from an eye-tracking study. Journal of Behavior Therapy and Experimental Psychiatry, 46, 107-114.

Epp, A. M., Dobson, K. S., Dozois, D. J., \& Frewen, P. A. (2012). A systematic metaanalysis of the Stroop task in depression. Clinical Psychology Review, 32, 316328.

Everaert, J., Koster, E. H., \& Derakshan, N. (2012). The combined cognitive bias hypothesis in depression. Clinical Psychology Review, 32, 413-424.

First, M. B., Spitzer, R. L., Gibbon, M., y Williams, J. B. W. (1995). Structured Clinical Interview for DSM-IV Axis I Disorders, Research Version. New York, NY: Biometrics Research, New York State Psychiatric Institute.

Fritzsche, A., Dahme, B., Gotlib, I. H., Joormann, J., Magnussen, H., Watz, H., ... \& von Leupoldt, A. (2010). Specificity of cognitive biases in patients with current depression and remitted depression and in patients with asthma. Psychological Medicine, 40, 815-826. 
Groves, S. J., Porter, R. J., Jordan, J., Knight, R., Carter, J. D., McIntosh, V. V. W., ... Joyce, P. R. (2015). Changes in neuropsychological function after treatment with metacognitive therapy or cognitive behavior therapy for depression. Depression and Anxiety, 32(6), 437-444.

Hilt, L. M., \& Pollak, S. D. (2013). Characterizing the ruminative process in young adolescents. Journal of Clinical Child \& Adolescent Psychology, 42, 519-530

Hofmann, S. G., Asmundson, G. J., \& Beck, A. T. (2013). The science of cognitive therapy. Behavior Therapy, 44, 199-212.

Kellough, J. L., Beevers, C. G., Ellis, A. J., \& Wells, T. T. (2008). Time course of selective attention in clinically depressed young adults: An eye tracking study. Behaviour Research and Therapy, 46, 1238-1243.

Lewinsohn, P. M., Antonuccio, D. O., Breckenridge, J. S., \& Teri, L. (1984). The 'Coping with Depression' course. Eugene, OR: Castalia Publishing Company.Leyman, L., De Raedt, R., Schacht, R., y Koster, H.W. (2007). Attentional biases for angry faces in unipolar depression. Psychological Medicine, 37, 393-402.

Lomas, T., Hefferon, K., \& Ivtzan, I. (2016). Positive developmental psychology: A review of literature concerning well-being throughout the lifespan (2016). The Journal of Happiness \& Well-Being, 4, 143-164.

Lopez-Gomez, I., Chaves, C., Hervas, G., \& Vazquez, C. (2017). Pattern of changes during treatment: a comparison between a positive psychology intervention and a cognitive behavioral treatment for clinical depression. The Spanish Journal of Psychology, 20. doi: 10.1017/sjp.2017.44. 
Lorenzo-Luaces, L., German, R. E., \& DeRubeis, R. J. (2015). It's complicated: The relation between cognitive change procedures, cognitive change, and symptom change in cognitive therapy for depression. Clinical Psychology Review, 41, 3-15. Lundqvist, D., Flykt, A., \& Öhman, A. (1998). The Karolinska Directed Emotional Faces (KDEF). Karolinska Hospital, Stockholm: Department of Neurosciences.

McCabe, S. B., Gotlib, I. H., \& Martin, R. A. (2000). Cognitive vulnerability for depression: Deployment of attention as a function of history of depression and current mood state. Cognitive Therapy and Research, 24, 427-444.

Moriana, J. A., Gálvez-Lara, M., \& Corpas, J. (2017). Psychological treatments for mental disorders in adults: a review of the evidence of leading international organizations. Clinical Psychology Review, 54, 29-43.

Muñoz, R.F., Aguilar-Gaxiola, S., \& Guzman, J. (1995). Group cognitive-behavioral therapy for major depression. Spanish version. Santa Monica, CA: Rand.

Peckham, A. D., McHugh, R. K., \& Otto, M. W. (2010). A meta-analysis of the magnitude of biased attention in depression. Depression and Anxiety, 27, 11351142 .

Roiser, J. P., Elliott, R., \& Sahakian, B. J. (2012). Cognitive mechanisms of treatment in depression. Neuropsychopharmacology, 37, 117-136.

Rømer Thomsen, K., Whybrow, P. C., \& Kringelbach, M. L. (2015). Reconceptualizing anhedonia: Novel perspectives on balancing the pleasure networks in the human brain. Frontiers in Behavioral Neuroscience, 9, 49. doi: 10.3389/fnbeh.2015.00049

Sánchez, A. \& Vázquez, C. (2013). Prototypicality and intensity of emotional faces using an anchor-point method. The Spanish Journal of Psychology, 16, 1-11. 
Sanchez, A., Duque, A., Romero, N., \& Vazquez, C. (2017). Disentangling the interplay among cognitive biases: evidence of combined effects of attention, interpretation and autobiographical memory in depression. Cognitive Therapy and Research, 41, $829-841$.

Sanchez, A., Vazquez, C., Gomez, D., \& Joormann, J. (2014). Gaze-fixation to happy faces predicts mood repair after a negative mood induction. Emotion, 14, 85-94.

Sánchez, A., Vázquez, C., Marker, C., Lemoult, J., \& Joormann, J. (2013). Attentional disengagement predicts stress recovery in depression: An eye-tracking study. Journal of Abnormal Psychology, 122, 303-313.

Segal, Z. V., \& Gemar, M. (1997). Changes in cognitive organization for negative selfreferent material following cognitive behavioural therapy for depression: A primed Stroop Study. Cognition and Emotion, 11, 501-516.

Shane, M., \& Peterson, J. B. (2007). An evaluation of early and late stage attentional processing of positive and negative information in dysphoria. Cognition and Emotion, 21, 789-815.

Sin, N. L., \& Lyubomirsky, S. (2009). Enhancing well-being and alleviating depressive symptoms with positive psychology interventions: A practice-friendly metaanalysis. Journal of Clinical Psychology, 65, 467-487.

Vazquez, C. (2017). What does positivity add to psychopathology? An Introduction to the Special Issue on "Positive emotions and cognitions in clinical psychology." Cognitive Therapy and Research, 41(3), 325-334.

Vazquez, C., Blanco, I., Sanchez, A., \& McNally, R. J. (2016). Attentional bias modification in depression through gaze contingencies and regulatory control using a new eye-tracking intervention paradigm: study protocol for a placebocontrolled trial. BMC Psychiatry, 16, 439. doi: 10.1186/s12888-016-1150-9 
Waechter, S., Nelson, A. L., Wright, C., Hyatt, A., \& Oakman, J. (2014). Measuring attentional bias to threat: Reliability of dot probe and eye movement indices. Cognitive Therapy and Research, 38, 313-333.

Watson, D., \& Naragon-Gainey, K. (2010). On the specificity of positive emotional dysfunction in psychopathology: Evidence from the mood and anxiety disorders and schizophrenia/schizotypy. Clinical Psychology Review, 30, 839-848.

Winer, E.S., \&Salem, T. (2016). Reward Devaluation: Dot-Probe Meta-Analytic Evidence of Avoidance of Positive Information in Depressed Persons. Psychological Bulletin, 142, 18-78.

Yiend, J., \& Mathews A. (2004). Biases in attention: Methods, mechanisms and meaning. In A. Wenzel \& D.C. Rubin (eds), Cognitive methods in clinical research. pp. 97-117. Washington, DC: APA Books.

Zinbarg, R. E., Mineka, S., Craske, M. G., Griffith, J. W., Sutton, J., Rose, R. D., ... \& Waters, A. M. (2010). The Northwestern-UCLA youth emotion project: Associations of cognitive vulnerabilities, neuroticism and gender with past diagnoses of emotional disorders in adolescents. Behaviour Research and Therapy, 48, 347-358. 
Table 1. Demographic and clinical characteristics

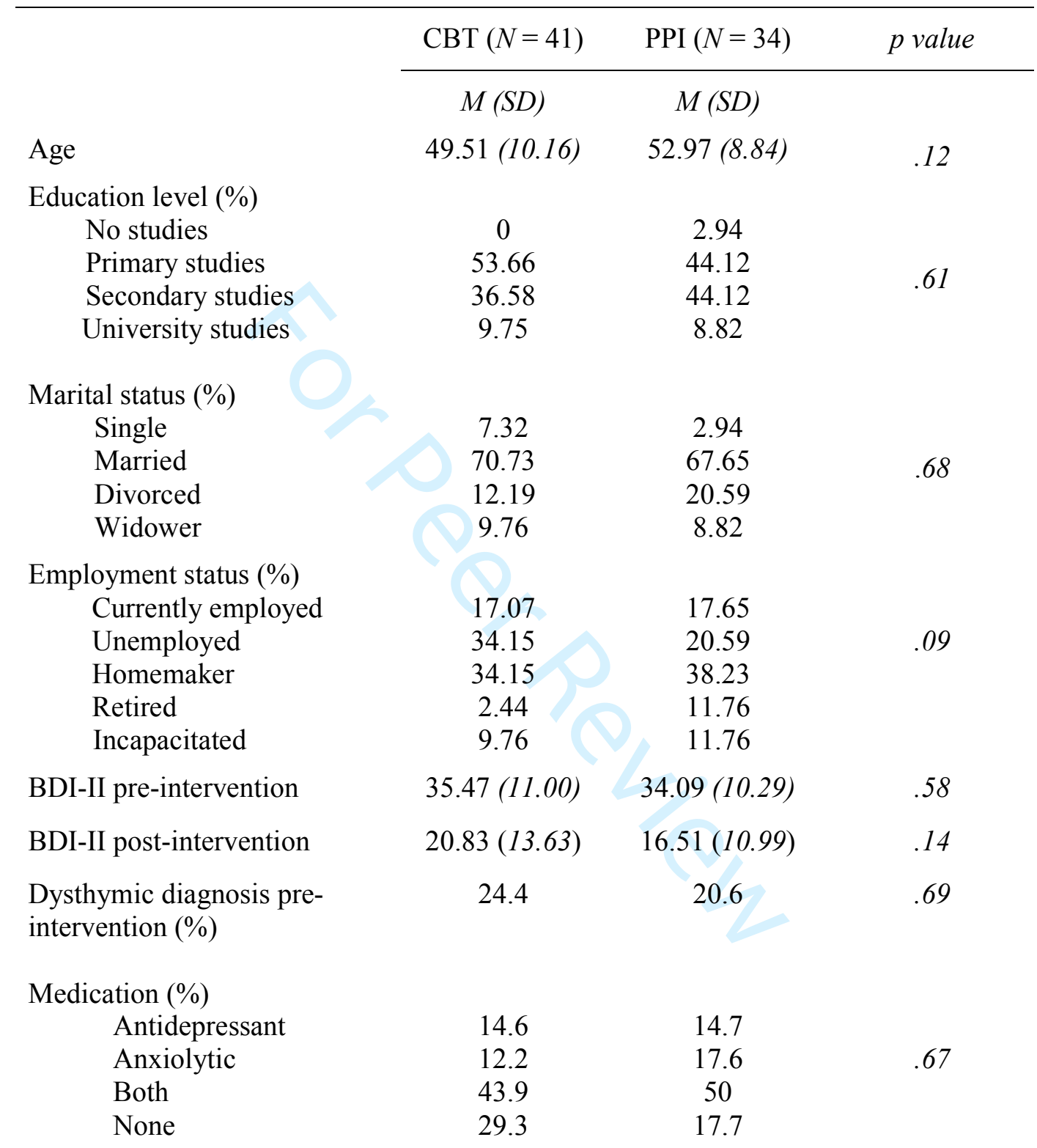

Note. CBT = Cognitive-Behavioral Therapy; PPI = Positive Psychology Interventions; $\mathrm{M}=$ Mean; $\mathrm{SD}=$ Standard Deviation; BDI-II = Beck Depression Inventory-II. 
Table 2. Means and standard deviations of the attentional indexes measured in all participants under two treatment condition for all experimental conditions (i.e., pairs of Happy-Neutral, Sad-Neutral, and Angry-Neutral faces).

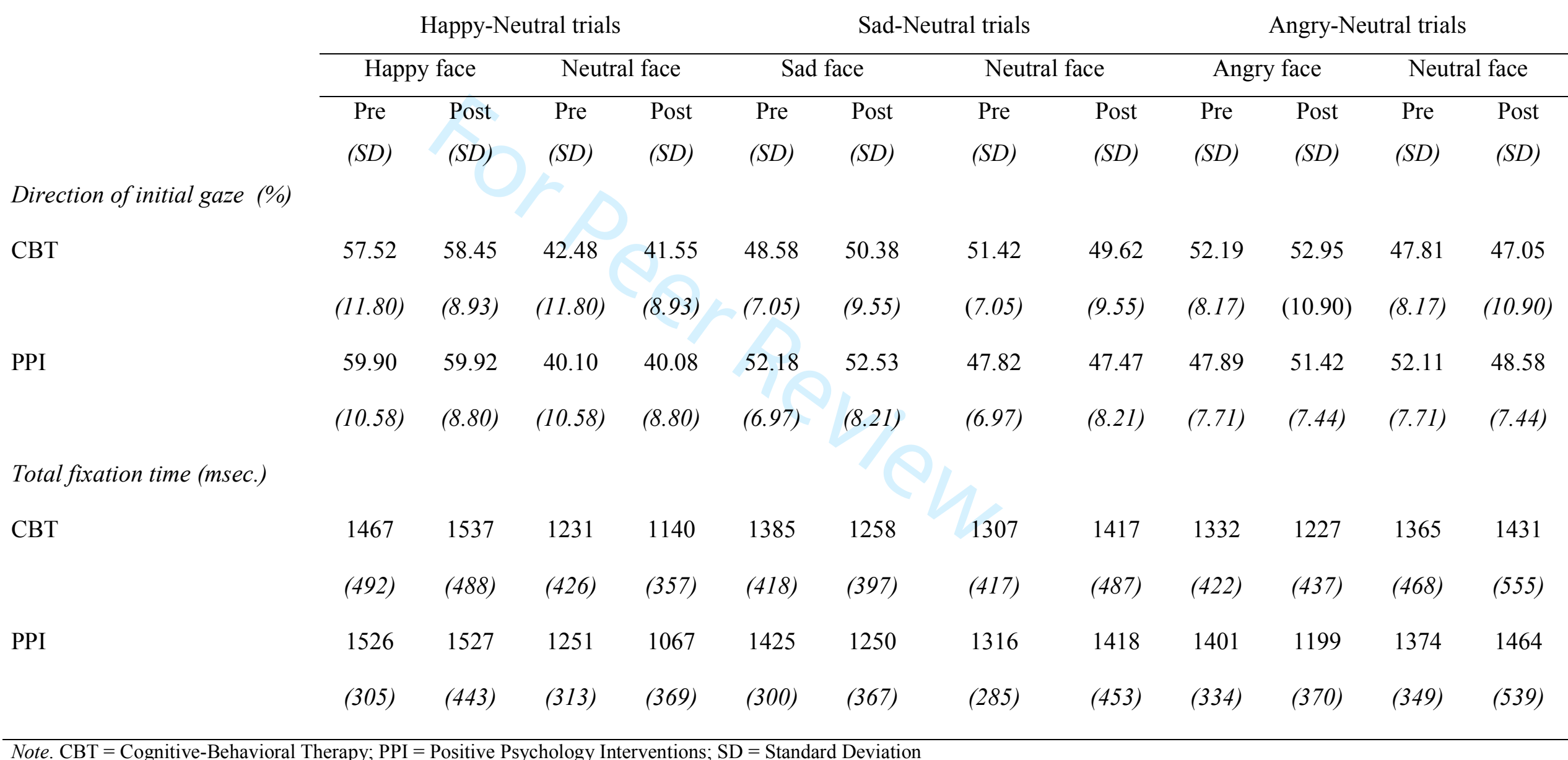


Table 3. Bivariate correlations between changes in attentional bias towards the emotional faces (i.e., happy, angry, sad) and changes in depressive symptoms before and after the interventions.

\begin{tabular}{ll}
\hline $\begin{array}{l}\text { Changes in attentional indexes } \\
\text { (Pre-post interventions) }\end{array}$ & $\begin{array}{l}\text { Changes in BDI-II } \\
\text { (Pre-post interventions) }\end{array}$ \\
\hline
\end{tabular}

Direction of initial gaze-Happy $\quad-.075$

Direction of initial gaze-Angry $\quad .005$

Direction of initial gaze-Sad $\quad-.014$

Total fixation time-Happy $\quad-.364 * *$

Total fixation time-Angry .250*

Total fixation time-Sad .266*

Note. ${ }^{*} \mathrm{p}<.05 ; * * \mathrm{p}<.01 . \mathrm{BDI}-\mathrm{II}=$ Beck Depression Inventory-II. 


\section{Figure legends}

Figure 1. Flow chart of the study design

Figure 2. Changes in depressive symptoms from pre-treatment to post-treatment.

Figure 3. Total fixation time changes from pre-treatment to post-treatment 


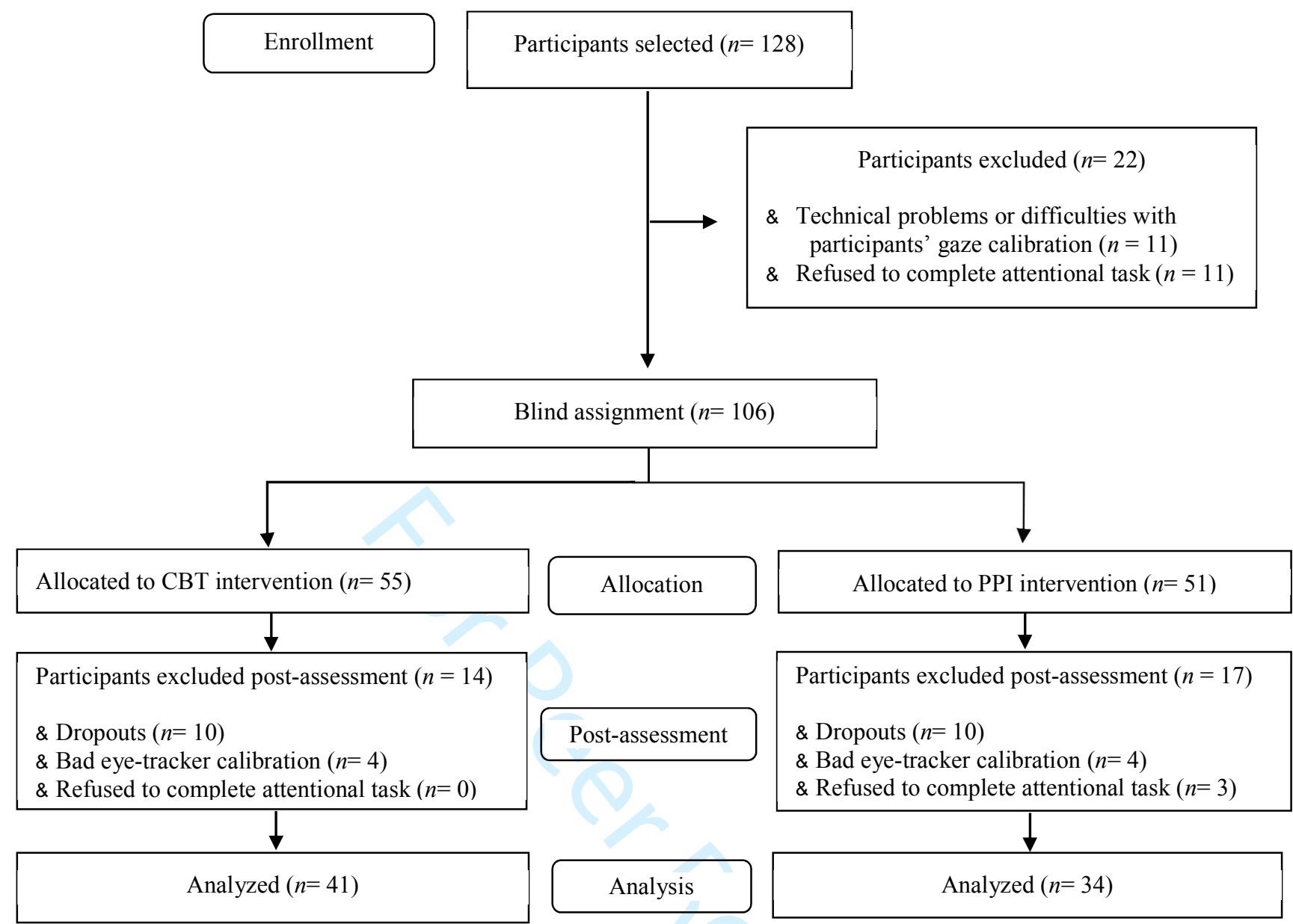

Figure 1. Flowchart of participants. CBT: cognitive-behavioral therapy, PPI: positive psychology intervention. 


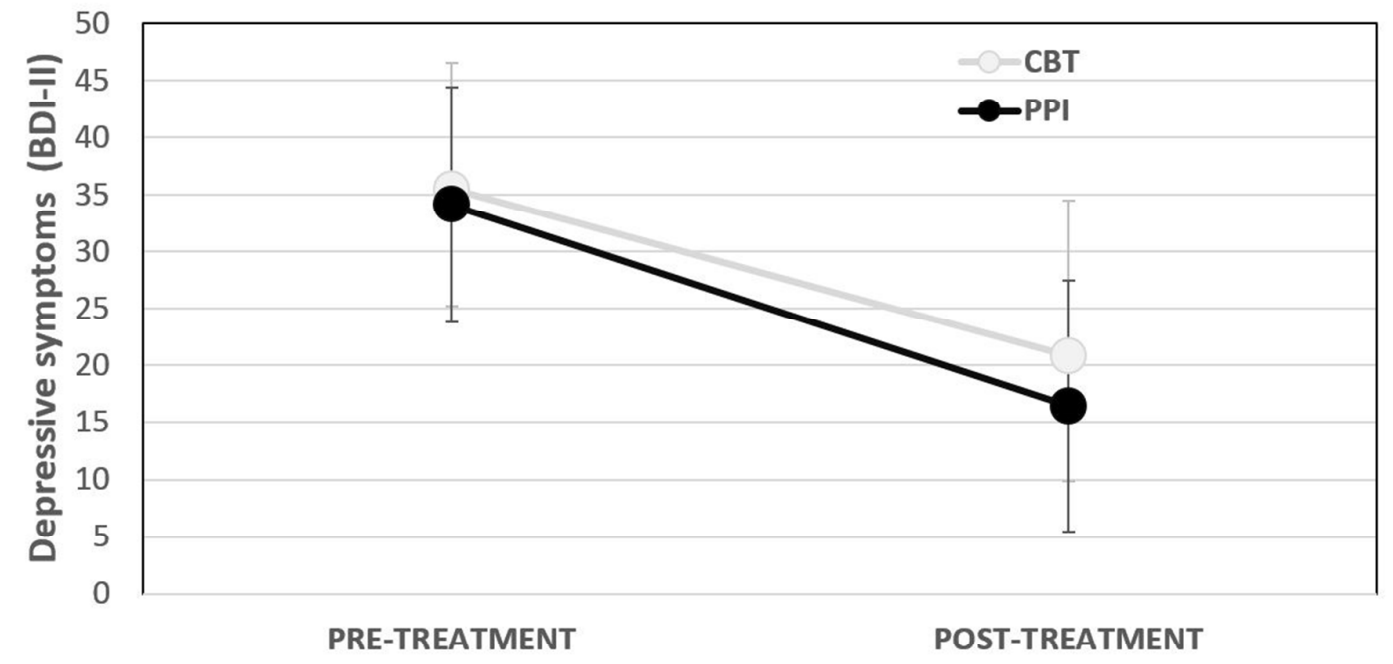

Note: $\mathrm{CBT}=$ Cognitive behavioral therapy; $\mathrm{PPI}=$ Positive psychology interventions 


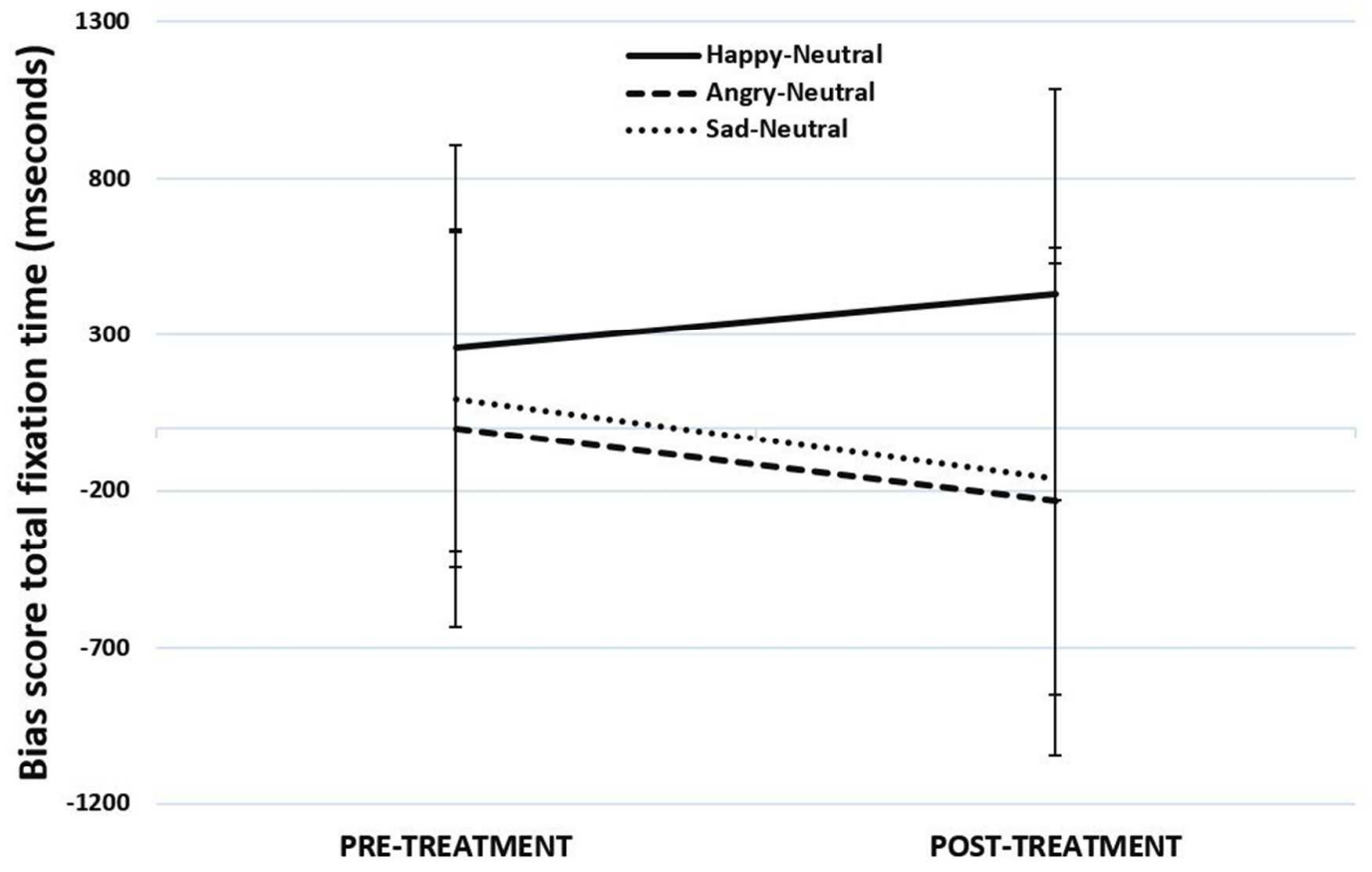

John Wiley \& Sons 